Design and performance of serial powered single-sided modules within an integrated stave assembly for the ATLAS tracker barrel upgrade

This article has been downloaded from IOPscience. Please scroll down to see the full text article.

2010 JINST 5 C12013

(http://iopscience.iop.org/1748-0221/5/12/C12013)

View the table of contents for this issue, or go to the journal homepage for more

Download details:

IP Address: 137.138.124.142

The article was downloaded on 11/07/2011 at 10:22

Please note that terms and conditions apply. 
Topical Workshop on Electronics for Particle Physics 2010, 20-24 SEPTEMBER 2010,

\title{
Design and performance of serial powered single-sided modules within an integrated stave assembly for the ATLAS tracker barrel upgrade
}

\author{
A. Affolder, ${ }^{1}$ J. Carrol, ${ }^{1}$ A. Greenall ${ }^{1,2}$ and M. Wormald \\ The University of Liverpool, Department of Physics, \\ Liverpool L697Z, United Kingdom \\ E-mail: ashley@liverpool.ac.uk
}

\begin{abstract}
The design and performance of prototype single-sided modules with ABCN-25 frontend chips and 10x10 $\mathrm{cm}^{2}$ Hamamatsu silicon strip sensors is presented. A low mass module assembly has been achieved by gluing a single-sided flex circuit, with read out chips, directly onto the sensor. The design exploits the embedded shunt regulation within the $\mathrm{ABCN}-25$ providing for a distributed and scalable powered architecture. This allows for multiple modules to be linked together serially to form larger stave structures of up to 12 modules. The stave's digital I/O is realised as a multi-drop LVDS bus flex cable glued to the stave core assembly using a custom receiver/transmitter ASIC (BCC). The results of preliminary electrical tests with 4 module stavelets will be presented.
\end{abstract}

KEYWORDS: Particle tracking detectors; Detector design and construction technologies and materials; Particle tracking detectors (Solid-state detectors)

\footnotetext{
${ }^{1}$ On behalf of the ATLAS Tracker Silicon Strip Upgrade Stave Programme

${ }^{2}$ Corresponding author.
} 


\section{Contents}

1 Introduction 1

1.1 The stave concept 1

1.2 Stave module overview 2

2 The stave flex hybrid $\quad 2$

2.1 Hybrid electrical performance 3

3 Stave module assembly $\quad 4$

3.1 Module electrical performance 4

3.2 Multiple module results 5

4 Conclusions 5

\section{Introduction}

The proposed ATLAS Tracker Upgrade for the SLHC will be all silicon with an inner pixel detector encompassed by a silicon micro-strip Barrel and End-Cap detector. The barrel consists of 3 inner short strip layers and 2 outer of long strip detectors. The most challenging is the short strip Barrel detector, consisting of 5184 modules with a silicon sensor area in excess of $51 \mathrm{~m}^{2}$. A R\&D programme has been setup to look at the development of short strip Barrel modules and their integration onto a stave structure. The key issues being addressed are the use of minimal material, mass production methods ( $>10000$ circuits will be required), a conservative assembly procedure (to facilitate distributed production at differing sites) and to be compatible with new proposed powering schemes (DC-DC or serial powering).

\subsection{The stave concept}

The concept is based on the principle of placing individual single-sided modules onto a lightweight, rigid structure. This is achieved by gluing completed modules onto a rectangular carbon fibre structure which has an embedded cooling circuit and Kapton bus cable glued to each face, the bus cable providing the module services (data I/O, powering and sensor bias). Shown conceptually in figure 1 , are a single module and a stave which consists of 24 modules (12 per side). A module is made with a $10 \mathrm{~cm}$ square micro-strip detector [1], to which are glued 2 Kapton flex (hybrid) circuits, each having 2 columns of $10 \mathrm{ABCN}-25$ readout ASICs [2]. For each circuit there is also a custom digital I/O ASIC (BCC ${ }^{1}-$ Basic Control Chip [3]) and a serial power protection board ${ }^{2}$ (PPB - Power Protection Board [4]).

\footnotetext{
${ }^{1}$ The BCC has AC-coupled LVDS receivers (clock and command), necessary for serial powering.

${ }^{2}$ Whilst serial powered, the PPB provides real time overvoltage protection and permits each hybrid to be individually bypassed under software control.
} 


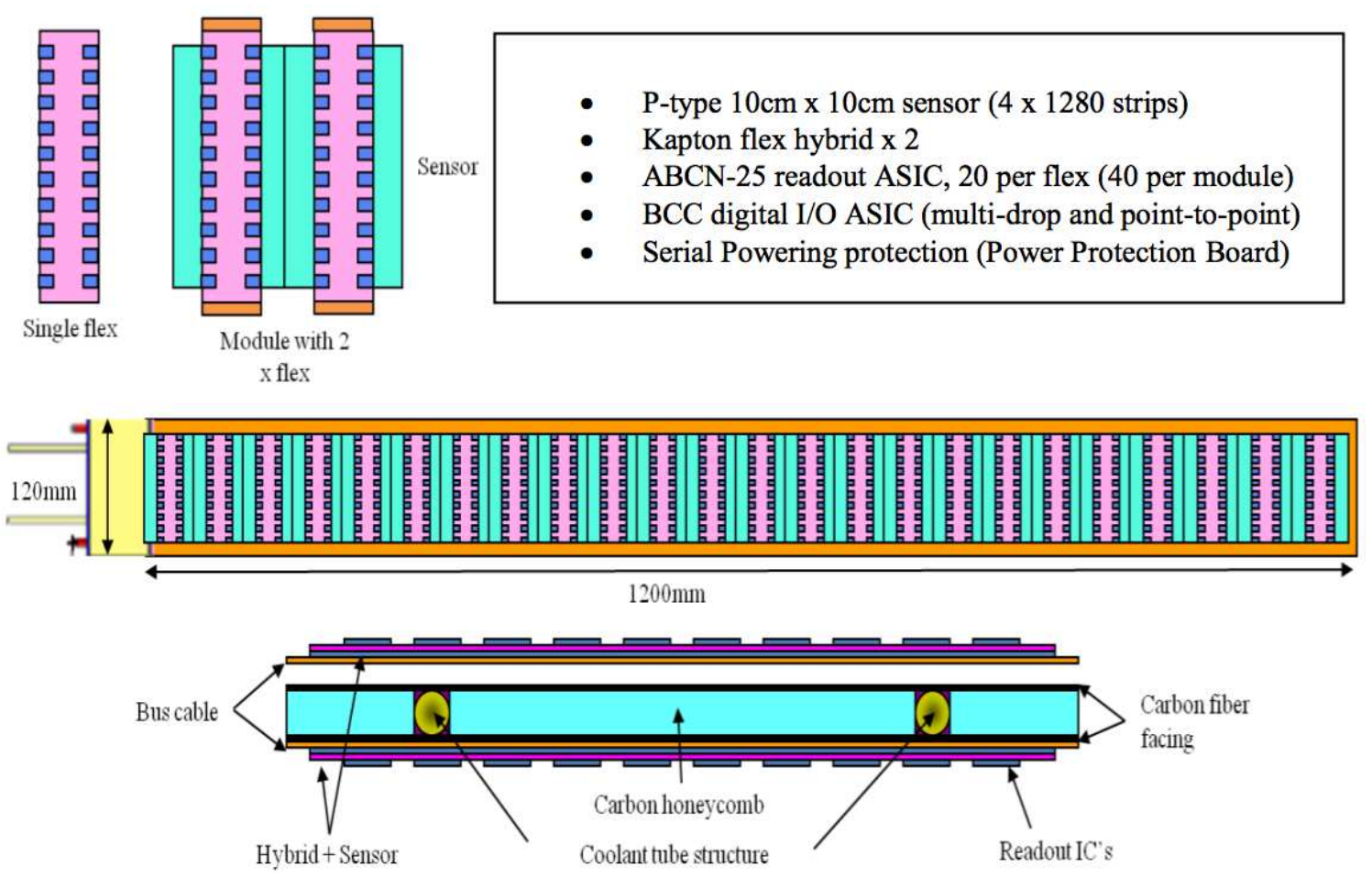

Figure 1. Single-sided module concept, the stave core and module integration onto a stave.

\subsection{Stave module overview}

The module design is driven by minimising material, this is achieved by attaching the readout ASICs to a low mass substrate-less flex circuit, which is then glued onto the sensor; providing both mechanical support and thermal management. All off circuit connections are made using wire bonds, no connectors are used. Further material reductions are achieved by the use of thin glue layers for both ASIC attachment and the hybrid flex gluing to the sensor and the removal of pitch adapters (direct ASIC-to-sensor wire bonding). This constrains the relative placement of the ASIC to sensor geometry to better than $80 \mu \mathrm{m}$ (approximately sensor strip pitch).

\section{The stave flex hybrid}

The flex is a five layer Kapton circuit which uses conservative design rules to optimise the manufacturer's volume and yield. The layout geometry is $100 \mu \mathrm{m}$ track and gap with blind vias $(150 \mu \mathrm{m}$ laser drilled with $375 \mu \mathrm{m}$ lands) using $50 \mu \mathrm{m}$ Kapton dielectrics for ease of handling during manufacture. The circuit has an asymmetric stack up of 4 active layers plus a shield i.e. Signal - Signal Power - Ground - Shield (top-down). The shield layer, sited closest to the sensor, has an optional single point contact to hybrid ground.

As a first step in evolving mass production methodologies, the flex is panelised, as shown in figure 2. The flex, composed of 8 sub-circuits, is selectively laminated to a FR4 substrate, with each sub-circuit laser cut on 4 sides with 4 retaining tabs, see figure 2 . The panelisation facilitates the auto-placement of passives and their solder-reflow. A further technique has also been developed 

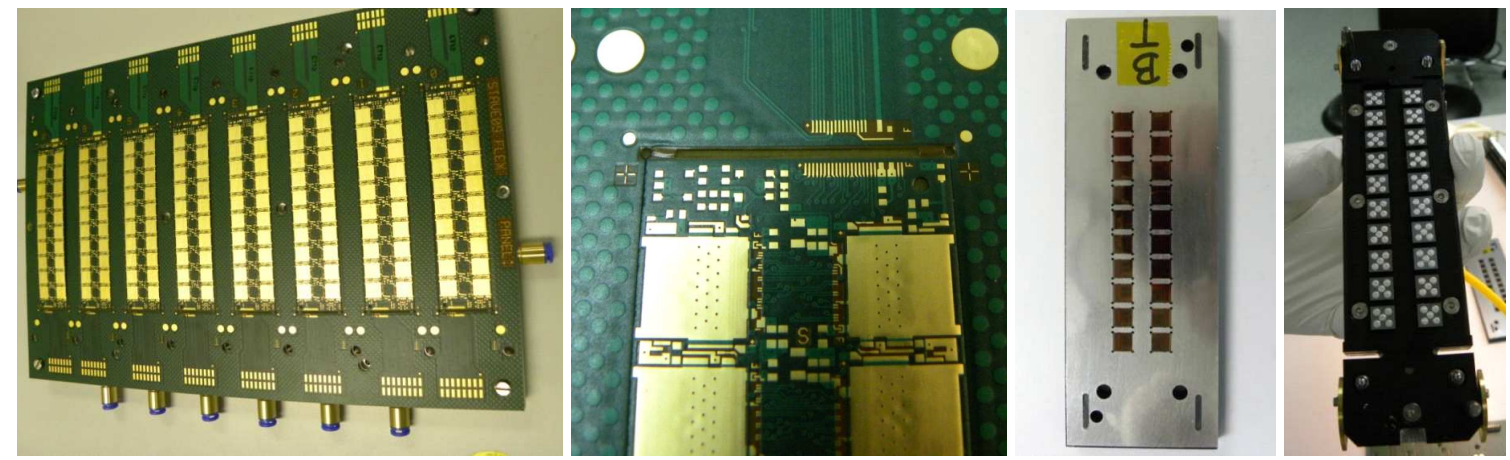

Figure 2. Panel of circuits, individual circuit detail, a chip tray with stencil attached and ASIC gluing.
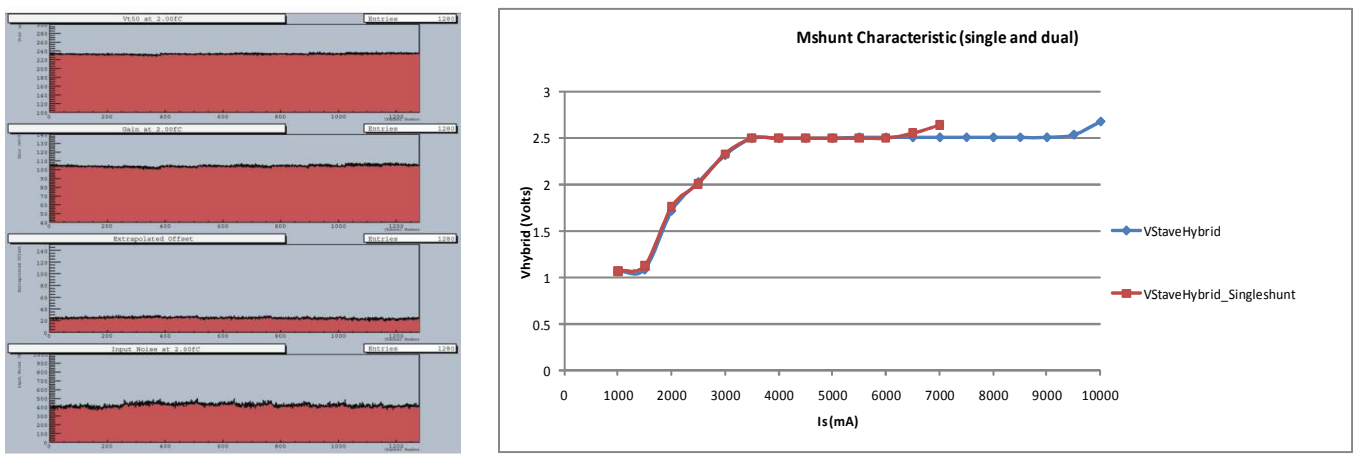

Figure 3. Typical Analogue and Shunt Regulation Characteristics of a hybrid.

for the mass attachment of 20 ASICs per sub-circuit, by using a 'chip-tray' to which is attached a $125 \mu \mathrm{m}$ thick laser cut stainless steel stencil (the geometry matching the ASIC lands on the hybrid), see figure 2. The ASICs are picked up in a single step and then have glue applied using another stencil (the stencil ensuring that a uniform glue layer is applied), again see figure 2. The ASICs are then attached to the sub-circuit in a single step.

The ASIC attachment technique results in a chip-to-chip placement to better than $15 \mu \mathrm{m}$ RMS and a uniform glue layer of approximately $80 \mu \mathrm{m}$ with a chip planarity of $<10 \mu \mathrm{m}$. The constraint on planarity is required as the hybrid pick up from a panel makes use of the chip faces and ensures that when attaching the hybrid to the sensor the glue layer is uniform.

\subsection{Hybrid electrical performance}

Hybrids, once completed, are tested in-situ on a panel. The circuit takes advantage of the features of the ABCN-25 chipset i.e. bi-directional data path readout and embedded distributed shunt regulation (a requirement for serial powering). The test sequence verifies the functional and electrical performance of the hybrid e.g. all bi-directional data paths are working, the shunts regulate to their specified $2.5 \mathrm{~V}$ and the analogue characterisation (input noise, gain and threshold variation) is as expected. A typical analogue response of a single column of $\mathrm{ABCN}-25 \mathrm{~s}$ is shown in figure 3 , indicating good threshold uniformity (after trimming), a gain of $110 \mathrm{mV} / \mathrm{fC}$ and the calculated input noise to be around 400e ENC. Also shown is the shunt regulation characteristic of the circuit, reg- 

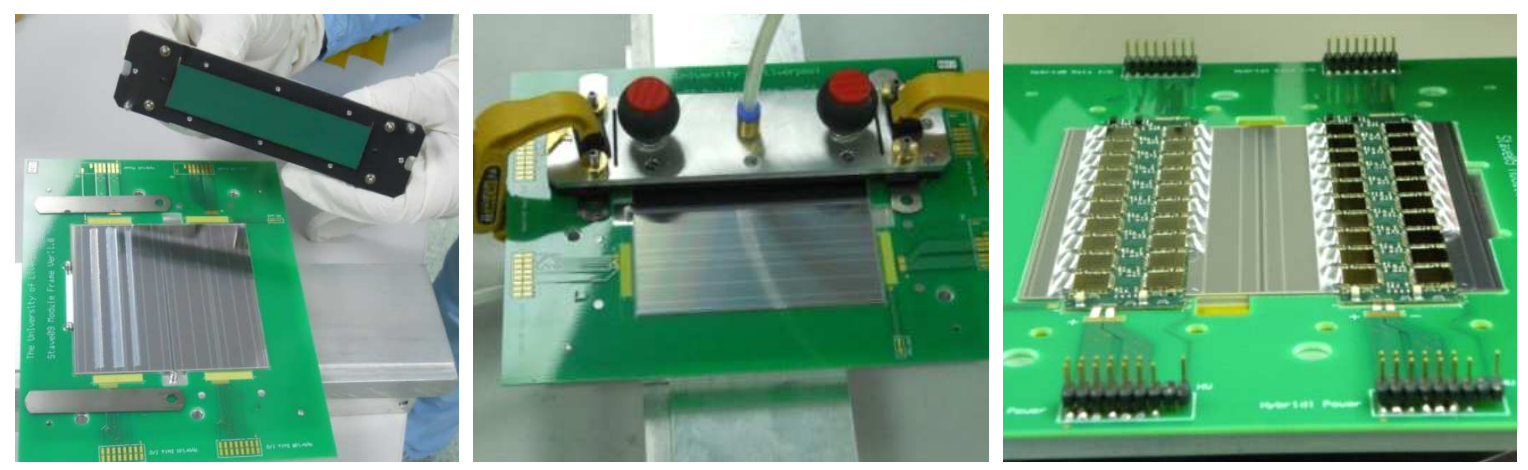

Figure 4. The sensor showing glue profile, hybrid attachment and a partly wire bonded module.

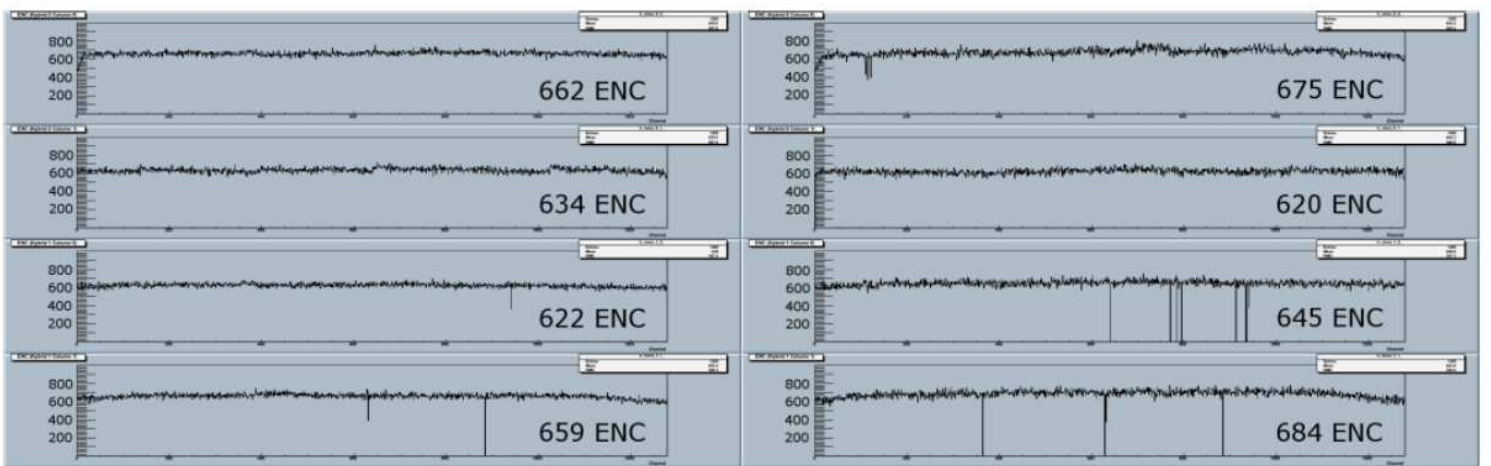

Figure 5. Module Input Noise per column for parallel (shown left) and serial powering (right).

ulating at the required $2.5 \mathrm{~V}$ with the ability to shunt up to a maximum of $9 \mathrm{~A}$ (normal operation is expected to be at $5 \mathrm{~A}$, shown by the vertical line).

\section{Stave module assembly}

After testing, a hybrid is detached from the panel by cutting its retaining tabs and then picked up using the ASIC attachment tooling. By using a jig (with precision dowel pins) plus a multifunction PCB frame, the sensor is positioned and has glue ${ }^{3}$ applied to its active surface (using an $80 \mu \mathrm{m}$ paper stencil). The hybrid is then attached to the sensor, then left to cure, before repeating the process with the second hybrid. A completed module (within the PCB frame), is then ready for wire bonding. Figure 4 shows the main module assembly steps.

\subsection{Module electrical performance}

A module can either be parallel (independent) or serially powered; when serially powered there exists a potential difference between adjacent hybrids of $\Delta \mathrm{V}=2.5 \mathrm{~V}$ ( $5 \mathrm{~V}$ across a module). Hence for a serially powered module, one hybrid must be AC referenced to the sensor, whilst both hybrids of a parallel powered module would be DC referenced.

Figure 5 shows the noise performance of all 4 columns from a module in both powering modes,

\footnotetext{
${ }^{3}$ Epolite FH-5313 (electrical grade epoxy) is used for attaching the hybrid to the sensor.
} 

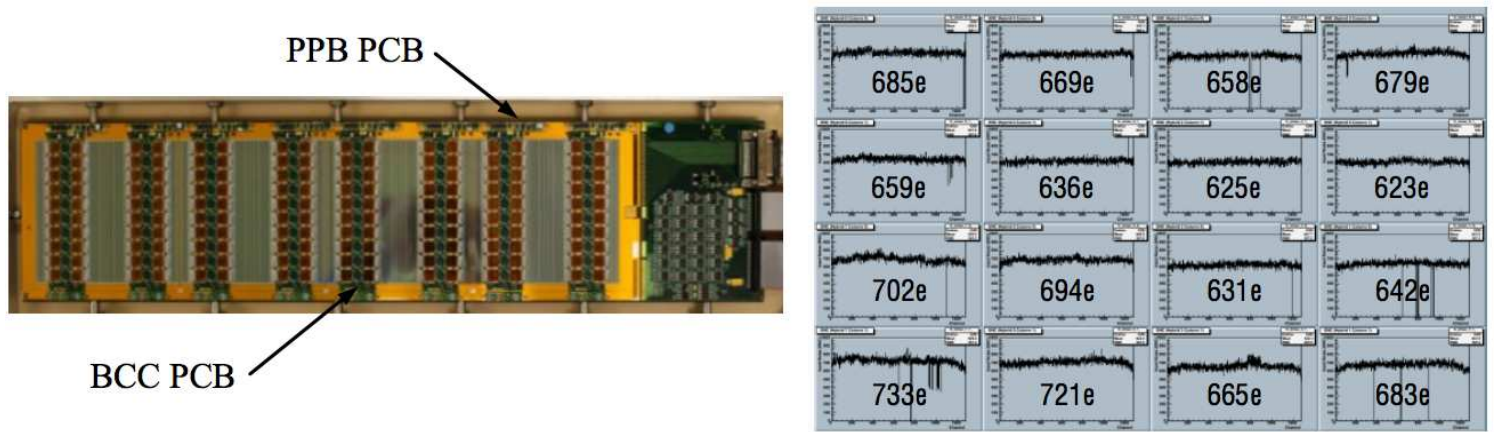

Figure 6. Photograph of a Stavelet with 4 modules mounted and the Module Noise for all channels.

the average noise of the serially powered module is comparable to that of the parallel configuration. In both instances, the module noise is typically $650 \mathrm{e}$ ENC cf. a design specification of $<750 \mathrm{e}$ RMS.

Further tests also revealed a noise feature common to all modules, irrespective of their powering, with the outer columns having typically $5 \%$ higher noise compared to the inner columns. A correlation has been found which attributes a noise contribution coming from the load capacitance presented to the ASIC front-ends from the hybrid ground/shield. This is due to the asymmetric attachment of the hybrids to the silicon sensor; the outer strips have greater hybrid coverage and hence coupling capacitance, resulting in these strips having higher noise.

\subsection{Multiple module results}

Multiple modules have been mounted onto a four module Stavelet [5] which will be used to evaluate differing grounding, powering and shielding options. The Stavelet is shown in figure 6, with PPB and $\mathrm{BCC}$ boards mounted along the edges of a row of modules. Also shown is a preliminary noise result for the Stavelet, running serially powered with non-optimised grounding and shielding configurations. The input noise is $\leqslant 750 \mathrm{e}$ ENC which compares reasonably well with the individual module performance.

\section{Conclusions}

Prototype short strip barrel modules have been successfully built using novel construction and assembly techniques whilst also taking on-board hybrid industrialization w.r.t. their assembly and testing. Furthermore the serial powering of modules, either singularly or in a chain, has been demonstrated to work with good electrical performance.

\section{Acknowledgments}

The authors wish to acknowledge colleagues at BNL, LBNL, Oxford University, Pennsylvania University, RAL, SLAC and UCL.

\section{References}

[1] Y. Unno et al., Silicon sensor development for the ATLAS upgrade for SLHC, Nucl. Instrum. Meth. A 569 (2006) 41. 
[2] F. Anghinolfi, Performance of the ABCN-25 readout chip for the ATLAS Inner Detector, in Topical Workshop on Electronics for Particle Physics TWEP09, Paris, France (2009).

[3] D. Lynn et al., Serial Power Protection for ATLAS Strip Staves, submitted to NIM (2010).

[4] M. Warren et al., see https://twiki.cern.ch/twiki/bin/viewauth/Atlas/BufferControlChip.

[5] J. Matheson, Progress and Advances in Serial Powering of Silicon Modules for the ATLAS Tracker Upgrade, in Topical Workshop on Electronics for Particle Physics 2010, 20-24 September 2010, Aachen, Germany. 